\title{
Associations of monoamine oxidase A gene first exon methylation with sexual abuse and current depression in women
}

\author{
David Checknita $^{1,2,5}$ - Tomas J. Ekström ${ }^{2} \cdot$ Erika Comasco $^{1} \cdot$ Kent W. Nilsson $^{1,3} \cdot$ Jari Tiihonen $^{2} \cdot$ Sheilagh Hodgins $^{2,4}$
}

Received: 1 November 2017 / Accepted: 8 March 2018 / Published online: 29 March 2018

(c) The Author(s) 2018

\begin{abstract}
Childhood physical abuse (PA) and sexual abuse (SA) interact with monoamine oxidase A (MAOA) gene polymorphism to modify risk for mental disorders. In addition, PA and SA may alter gene activity through epigenetic mechanisms such as DNA methylation, thereby further modifying risk for disorders. We investigated whether methylation in a region spanning the $M A O A$ first exon and part of the first intron was associated with PA and/or SA, MAOA genotype, alcohol dependence, drug dependence, depression disorders, anxiety disorders, and conduct disorder. 114 Swedish women completed standardized diagnostic interviews and questionnaires to report PA and SA, and provided saliva samples for DNA extraction. DNA was genotyped for MAOA-uVNTR polymorphisms, and methylation of a MAOA region of interest (chrX: 43,515,544-43,515,991) was measured. SA, not PA, was associated with hypermethylation of the MAOA first exon relative to no-abuse, and the association was robust to adjustment for psychoactive medication, alcohol and drug dependence, and current substance use. SA and MAOA-uVNTR genotype, but not their interaction, was associated with MAOA methylation. SA associated with all measured mental disorders. Hypermethylation of $M A O A$ first exon mediated the association of SA with current depression, and both methylation levels and SA independently predicted lifetime depression. Much remains to be learned about the independent effects of SA and MAOA-uVNTR genotypes on methylation of the MAOA first exon.
\end{abstract}

Keywords Child abuse $\cdot$ Depression $\cdot$ Epigenetics $\cdot$ Gene-environment interaction methylation $\cdot$ Women

Electronic supplementary material The online version of this article (https://doi.org/10.1007/s00702-018-1875-3) contains supplementary material, which is available to authorized users.

David Checknita

drchecknita@gmail.com

1 Department of Neuroscience, Uppsala University, Uppsala, Sweden

2 Department of Clinical Neuroscience, Karolinska Institute, Stockholm, Sweden

3 Centre for Clinical Research, Västmanland County Council, Uppsala University, Västerås, Sweden

4 Institut Universitaire en Santé Mentale de Montréal, Université de Montréal, Montreal, Canada

5 Karolinska Universitetssjukhuset, Psychiatry Building R5:00 c/o Jari Tiihonen, 17176 Stockholm, Sweden

\section{Introduction}

The prevalence of childhood physical abuse (PA) and sexual abuse (SA) in the US is estimated at 28.4 and $4.5 \%$, respectively (Hussey et al. 2006). Smaller proportions of girls than boys experience PA (Thompson et al. 2004) and greater proportions experience SA (Singh et al. 2014). Metaanalyses indicate that childhood PA and SA are associated with greatly increased risks for conduct disorder (CD) in childhood, antisocial personality disorder (ASPD) in adulthood, and substance use, depression, and anxiety disorders (Hussey et al. 2006; Springer et al. 2007; Fergusson et al. 2008; Singh et al. 2014; Li et al. 2016; Rita et al. 2016); all disorders associated with serotonergic dysfunction (Comai et al. 2012; Booij et al. 2015). Both PA and SA are associated with abnormalities of gray and white matter brain structures (McCrory et al. 2010), and activity (Herringa et al. 2013; Teicher and Samson 2016), particularly in limbic and hippocampal structures which are targets of serotonergic projections. Furthermore, childhood maltreatment modifies the association of genetic polymorphisms involved in the 
regulation of brain serotonin with antisocial behaviour (Pavlov et al. 2012) and depression (Mandelli and Serretti 2013).

Within the promoter region of the MAOA gene, which encodes the monoamine oxidase (MAO) enzyme that metabolizes serotonin and other monoamine neurotransmitters, there is a 30 base-pair functional variable number of tandem repeats polymorphism (MAOA-uVNTR) including short (MAOA-S) and long (MAOA-L) variants associated with low expression and high expression, respectively, in cell cultures (Sabol et al. 1998; Beach et al. 2010).

The expression of MAOA may be altered by environmental factors through epigenetic mechanisms. A region of interest (ROI) within the MAOA promoter spanning the first exonic and intronic regions was previously identified and characterized as a putative locus for methylation (Shumay and Fowler 2010). Methylation of this region was negatively correlated with brain MAO enzymatic activity in healthy men (Shumay et al. 2012). Male offenders with ASPD showed hypermethylation in this region relative to healthy men, which was associated with whole-blood serotonin levels among offenders and reduced transcriptional activity in vitro (Checknita et al. 2015). However, it is not known if hypermethylation is specifically associated with ASPD or with maltreatment that is common among men with ASPD (Hill and Nathan 2008), or substance use disorders that characterize almost all men with ASPD (Goldstein et al. 2017), or anxiety disorders presented by approximately half of male offenders with ASPD (Hodgins et al. 2010), or depression that is also elevated in this population (Lenzenweger et al. 2007). Hypomethylation of this region was observed among women with depression (Melas et al. 2013; Melas and Forsell 2015) and those with panic disorder relative to healthy women (Ziegler et al. 2016). Stressful life events similarly associate with depression and anxiety (McCrory et al. 2012). Hypermethylation of MAOA exon 1 was also observed among women relative to men, suggestive of X-chromosome inactivation given the gene's location on the X-chromosome (Melas and Forsell 2015). It is not presently known whether PA and SA are associated with $M A O A$ promoter methylation.

Differentially methylated regions in candidate genes associated with mental disorders are often located in regions flanking transcription start sites and typically do not overlap with known functional polymorphisms in the same gene promoters (Dammann et al. 2011; Checknita et al. 2015). It is presently not known whether genotype and interactions of genotype and environmental factors are associated with altered methylation. One study showed that lower promoter methylation levels of the serotonin transporter gene (5HTT) were associated with cortisol responses to a stress test only among carriers of the low-expressing variant of the 5HTTLPR promoter polymorphism (5HTT-S) (Alexander et al. 2014). Among adult carriers of 5HTT-S, childhood PA was associated with reduced $5 H T T$ mRNA levels, though methylation was not involved (Alexander et al. 2014). It is not currently known if $M A O A$ genotype interacts with childhood maltreatment to determine methylation levels in the $M A O A$ ROI.

Additional evidence that environmental factors lead to epigenetic changes, and that the magnitude of the changes depends on genotype, would have implications for treatment. For example, among women with panic disorder who responded positively to cognitive-behavioural therapy, methylation levels rose to equal those observed among healthy women, while no significant change in methylation was detected among treatment non-responders (Ziegler et al. 2016). Although the role of MAOA-uVNTR genotype in treatment response is not fully understood, recent studies suggest that men and women carrying MAOA-S, as compared to MAOA-L carriers, display greater reductions in symptoms of depression when taking mirtazapine (Tzeng et al. 2009) and of panic disorder following cognitive-behavioural treatment (Reif et al. 2014).

The present study examined methylation of an ROI within the $M A O A$ gene promoter flanking the first exonic region in DNA derived from saliva samples of young women, most of whom had displayed antisocial behaviour in adolescence, and one-half of whom had experienced PA and/or SA. The first aim was to determine whether PA and/or SA were associated with methylation in the MAOA ROI, and whether any observed associations were due to lifetime or current psychoactive medication, lifetime or current alcohol or drug dependence, current alcohol or drug use, each of which is common among persons who have experienced PA and/or SA, each of which has been shown to be associated with methylation levels in MAOA ROI and/or wide-spread epigenetic alterations across the genome (Philibert et al. 2008; Nestler 2014; Booij et al. 2015; Szyf 2015; Ziegler et al. 2016). The second aim was to determine whether associations between abuse and methylation differed by $M A O A$ genotype. The third aim was to determine whether methylation of the MAOA ROI mediates associations of abuse with alcohol dependence, drug dependence, depression disorders, anxiety disorders, and $\mathrm{CD}$.

\section{Method}

\section{Participants}

The sample included 114 Swedish women aged on average 22 years, 91 were recruited at a clinic for adolescent substance misuse, 75 of whom sought treatment and 16 of whom were non-biological siblings of other clinic attendees (not biologically related to any other participant), and 23 healthy women. The 75 ex-clients completed structured, 
validated, diagnostic interviews and questionnaires to report on PA and SA at first contact with the clinic and 6, 12, 60, and 75 months later. At the 60-month follow-up, the 16 non-biologically related siblings completed the same assessments, and all 91 participants provided saliva samples for DNA extraction. At the 75-month follow-up, the healthy female participants matched on age to other participants were recruited, completed assessments similar to those completed by the other participants, and provided saliva for DNA extraction.

\section{Measures}

\section{DNA}

Genomic DNA was extracted with a standard in-silica-based method from saliva samples collected with the Oragene SelfCollection Kit (DNA Genotek Inc. Ottawa, ON, Canada) according to the manufacturer's guidelines. Saliva samples were collected during the meeting between the participant and the researchers, using the Oragene self-collection kit (DNA Genotek ${ }^{\circledR}$, Canada: http://www.dnagenotek.com). The samples were stored at room temperature in accordance with the manufacturer's guidelines. Genomic DNA was extracted from $200 \mu \mathrm{l}$ of saliva using the silica-based Kleargene DNA extraction method (LGC ${ }^{\circledR}$, UK: http://www.lgcgroup.com) and stored at $-20^{\circ} \mathrm{C}$, and then $-80{ }^{\circ} \mathrm{C}$, prior to genotyping procedures and methylation analysis of the MAOA ROI. Genotyping and methylation analyses were performed in a blinded manner.

\section{Genotypes}

Genotyping was performed using a standard PCR technique, followed by gel electrophoresis. The target 30-bp repeat target-region of MAOA (MAOA-uVNTR) was amplified using forward primer $5^{\prime}$ ACA GCC TGA CCG TGG AGA AG $3^{\prime}$ and reverse primer 5' GAA CGG ACG CTC CAT TCG GA 3' (Sabol et al. 1998). In accordance with prior in vitro functional analyses of the MAOA-uVNTR (Beach et al. 2010), the 3 repeat variant was defined as the short (MAOA-S) allele, and 3.5, 4, or 5 repeat variants as the long (MAOA-L) allele. Genotype prevalence is presented in Table 1. Hardy-Weinberg Equilibrium was verified for

Table 1 Characteristics of participants carrying different $M A O A$ genotypes

\begin{tabular}{|c|c|c|c|c|}
\hline & \multicolumn{3}{|c|}{ MAOA-uVNTR $^{\mathrm{a}}(n=114)$} & \multirow[t]{2}{*}{ Statistics } \\
\hline & SS & SL & LL & \\
\hline Participants & $n=20$ & $n=51$ & $n=43$ & \\
\hline$\%$ Participants & 17.5 & 44.7 & 37.7 & \\
\hline Mean (SD) age at saliva collection & $22.56(3.95)$ & $22.00(3.31)$ & $22.38(3.13)$ & ns \\
\hline \multicolumn{5}{|l|}{ Physical and sexual abuse } \\
\hline$\%(n=)$ physical and/or sexual abuse & $45.0(9)$ & $64.7(33)$ & $53.5(23)$ & ns \\
\hline$\%(n=)$ physical abuse only & $8.3(1)$ & $18.2(4)$ & $16.7(4)$ & ns \\
\hline$\%(n=)$ sexual abuse only & $21.4(3)$ & $43.8(14)$ & $31.0(9)$ & ns \\
\hline \multicolumn{5}{|l|}{ Lifetime diagnoses } \\
\hline$\%(n=)$ alcohol dependence & $10.0(2)$ & $33.3(17)$ & $25.6(11)$ & ns \\
\hline$\%(n=)$ drug dependence & $25.0(5)$ & $23.5(12)$ & $30.2(13)$ & ns \\
\hline$\%(n=)$ anxiety disorders & $40.0(8)$ & $68.6(35)$ & $51.2(22)$ & ns \\
\hline$\%(n=)$ depression disorders & $55.0(11)$ & $68.6(35)$ & $51.2(26)$ & ns \\
\hline$\%(n=)$ conduct disorder before 15 & $30.0(6)$ & $41.2(21)$ & $37.2(16)$ & ns \\
\hline \multicolumn{5}{|l|}{ Current diagnoses } \\
\hline$\%(n=)$ alcohol dependence & $5.0(1)$ & $19.6(10)$ & $14.0(6)$ & ns \\
\hline \multirow[t]{3}{*}{$\%(n=)$ drug dependence } & $5.0(1)$ & $11.8(6)$ & $20.9(9)$ & ns \\
\hline & \multicolumn{3}{|c|}{ MAOA-uVNTR $^{\mathrm{a}}(n=143)$} & \\
\hline & SS & SL & LL & \\
\hline$\%(n=)$ anxiety disorders & $20.0(4)$ & $62.7(32)$ & $44.2(19)$ & $\begin{array}{l}\chi^{2}(2, \\
\quad N=114)=10.97 \\
p=0.004\end{array}$ \\
\hline$\%(n=)$ depression disorders & $45.0(9)$ & $43.1(22)$ & $32.6(14)$ & ns \\
\hline
\end{tabular}

$S D$ standard deviation, $L L$ homozygous for the long alleles, $S L$ heterozygous, $S S$ homozygous for the short alleles, $n s$ not significant

${ }^{a}$ Monoamine oxidase A variable number of tandem repeats 
MAOA-uVNTR genotype using an $X^{2}$ test $(p=0.47)$. Allelic frequencies of MAOA-uVNTR in our study were consistent with the results of previous studies of Swedish females (Åslund et al. 2013).

\section{Methylation analysis of $M A O A$ promoter region of interest}

Methylation analysis targeted a previously characterized 448bp region of interest (hg $19 \mathrm{chrX}$ : 43,515,544-43,515,991) within the MAOA promoter comprised of $16 \mathrm{CpGs}$ spanning the first exon and part of the first intronic region (Checknita et al. 2015). Genomic DNA extracted from saliva was first bisulfite-treated using EZ DNA Methylation ${ }^{\mathrm{TM}}$ Kit (Zymo Research Corporation, Irvine, $\mathrm{CA}$ ) and then assayed using Agena Bioscience's EpiTYPER at Karolinska University Hospital Mutation Analysis Core Facility (MAF). Resulting data represented the percentage of methylation at each $\mathrm{CpG}$ to the nearest $0.5 \%$. CpGs were denoted numerically based on their 5' $-3^{\prime}$ position within the ROI based on the forward strand genomic sequence. To ensure optimal technical outcomes, an amplicon designed on the reverse strand covered CpGs 1-13 and another amplicon covering CpGs 13-16 was designed on the forward strand. For additional information regarding the EpiTYPER procedure and quality control, please refer to Online Resource 1.

\section{Physical abuse}

Participants completed the revised Conflict Tactics Scales (Straus et al. 1996) to report on physical abuse (PA) by parents. Responses were dichotomously coded as absent or present (hit with fist or kicked hard, hit on part of body other than bottom with hard object, thrown or knocked down, grabbed around neck and choked, hit repeatedly very hard, burned, or threatened with a gun or knife).

\section{Sexual abuse}

Sexual abuse (SA) was assessed using items from the Sexual Experience Survey (Koss and Oros 1982), the Sexual and Physical Abuse Questionnaire (Kooiman et al. 2002), and McArthur Community Violence Instrument (Steadman et al. 1998). SA was dichotomously coded as absent or present if any of the following were reported: forced to have sex against her will by a person in authority, by offering alcohol or drugs, or by physical violence.

\section{Lifetime and current mental disorders}

All participants completed the Structured Clinical Interview for DSM-IV, both I and II (SCID I, SCID II) at DNA collection. Lifetime and current (past 6 months) diagnoses of alcohol dependence, drug dependence, depression disorder (major depression disorder, dysthymia, depression disorder not-otherwise-specified, or substance-induced mood disorder), anxiety disorder (agoraphobia, generalized anxiety disorder, anxiety disorder not-otherwise-specified, obsessive compulsive disorder, panic disorder, post-traumatic stress disorder, social phobia, specific phobia, or substanceinduced anxiety disorder), and conduct disorder (CD), prior to age 15 , were recorded. Thus, $\mathrm{CD}$ and lifetime diagnoses were made prior to DNA collection (baseline, 6-, 12-, and 60-months follow-ups) for 91 participants and reconfirmed at the time of the DNA collection when the current diagnoses were made. For the other 23 participants, lifetime and current diagnoses were made when DNA was collected.

\section{Current alcohol and drug use}

At the time of DNA collection, participants completed the 10-item AUDIT questionnaire (Babor et al. 2001) reporting number of drinks consumed, frequency of drinking, frequency of heavy drinking, alcohol dependence symptoms, and alcohol-related problems during the past 12 months. Responses were summed to obtain a total score ranging from 0 to 40 . A higher score indicated more alcohol-related problems. In addition, at the time of DNA collection, participants completed the 11-item DUDIT questionnaire (Berman et al. 2005) on report frequency of drug use, frequency of heavy drug use, polydrug use, drug dependence symptoms, and drug-related problems during the past 12 months. Responses were summed to obtain a score ranging from 0 to 44 . A higher score indicated more drug-related problems.

\section{Psychoactive medication use}

At all waves of data collection, participants reported use of psychoactive medications including stimulants, hypnotics, anxiolytics, antidepressants, antipsychotics. Lifetime and current (past 6 months) use of psychoactive medication was coded present or absent.

\section{Statistical analyses}

Chi-square and one-way ANOVAs were computed to compare characteristics of carriers of MAOA-uVNTR genotypes. To explore associations between abuse and MAOA ROI methylation levels, two-way mixed-model ANOVAs with post hoc LSD for multiple comparisons were computed. These models were used to test group differences in mean overall ROI methylation and at each CpG comparing: (1) 65 participants who had experienced PA and/or SA to 49 non-abused (NA) participants who experienced neither PA nor SA. Power analyses were calculated for each of the three comparisons using average group differences in ROI methylation (mean 2.56, SD 4.27) from (Checknita et al. 2015): PA and/or SA 
versus NA included 114 participants and provided 0.97 power to detect a similar group difference at an alpha of 0.05 ; PA only versus NA included 58 participants providing 0.78 power; and SA versus NA included 75 participants with 0.87 power. One-way ANCOVAs were computed to determine if associations between maltreatment and $M A O A$ ROI methylation were robust to adjustments for lifetime and current use of psychoactive medications (stimulants, hypnotics, anxiolytics, antidepressants, and antipsychotics), lifetime and current diagnoses of alcohol dependence, current alcohol use, lifetime and current diagnoses of drug dependence, and current drug use.

Next, general-linear regression models (GLMs) were computed to examine associations of $M A O A$ genotype, abuse, and interactions of genotype and abuse with methylation at each locus and mean methylation of exon 1 . The model included three predictors: $M A O A$ genotype, $\mathrm{SA}$, and the interaction of $M A O A$ genotype and SA.

Chi-square analyses were used to examine associations of abuse with lifetime and current diagnoses of mental disorders. Step-wise logistic regression models were computed to determine whether methylation levels at each locus mediated associations between abuse and diagnoses. Abuse was entered into step 1; methylation was entered in step 2; and in step 3, both abuse and methylation were entered. If abuse was significantly associated with a diagnosis in step 1 , abuse was significantly associated with methylation in step 2, and only methylation was associated in step 3, results were interpreted to suggest that methylation fully mediated the association between abuse and the clinical diagnosis. To verify if mediation effects of methylation differed significantly from the direct associations of SA and diagnoses, PROCESS for SPSS v2.15 was used with the bootstrapping procedure for indirect effects described by Hayes (2013).

\section{Results}

As presented in Table 1, neither age nor proportions of participants with PA and/or SA, PA, SA, lifetime diagnoses, or $\mathrm{CD}$ before age 15 varied by MAOA genotype. Proportionately, more of the MAOA-SL carriers, than SS or LL, presented, at a trend, lifetime diagnoses of anxiety disorders. Current diagnoses varied by genotype: proportionately more MAOA-SL carriers, than SS or LL, presented anxiety disorders. Characteristics of participants that were entered into analyses as covariates are presented on Online Resource Table S1.

\section{Are physical and sexual abuse associated with MAOA promoter ROI methylation?}

\section{$\mathrm{PA}$ and/or SA}

A two-way mixed-model ANOVA revealed a significant main effect of group $[F(1,204)=13.622, p<0.001]$ indicating higher methylation among those who had experienced PA and/or SA compared to NA. Post hoc LSD analysis revealed significantly higher methylation levels among those who experienced PA and/or SA compared to NA at CpG sites $2 / 3(p<0.001), 4(p<0.001), 5 / 6(p<0.001)$, $7 / 8(p<0.001), 10(p<0.001)$, and $11(p=0.002)$. Lower methylation among those who experienced PA and/or SA compared to NA was found at $\mathrm{CpG15}(p=0.036)$. One-way ANCOVAs indicated that these associations were robust to adjustments for lifetime and current use of psychoactive medications, lifetime and current diagnosis of alcohol dependence and current alcohol use, and lifetime and current drug dependence and current drug use.

\section{PA only}

A two-way ANOVA revealed a significant main effect of group $[F(1,115)=4.951, p=0.028]$ indicating higher methylation among those who had experienced PA compared to NA. Post hoc LSD analysis revealed significantly higher methylation levels among those who had experienced PA at $\mathrm{CpG}$ site $7 / 8(p=0.023)$. Only nine participants experienced PA only. Therefore, these results were not adjusted for covariates.

\section{SA only}

A two-way ANOVA revealed a significant main effect of group $[F(1,187)=12.693, p<0.001]$ indicating higher overall ROI methylation levels among participants who had experienced SA compared to those with NA. Post hoc LSD revealed significantly higher methylation levels among the SA group compared to NA which were again specific to MAOA first exon CpG sites $2 / 3(p<0.001), 4(p=0.001), 5 / 6$ $(p<0.001), 7 / 8(p=0.001)$, and $11(p=0.002)$. Lower methylation among those who experienced SA only compared to NA was found at CpG15 $(p=0.018)$. Results are illustrated in Fig. 1. One-way ANCOVAs revealed that these associations remained robust after adjusting for all covariates.

\section{Does sexual abuse interact with MAOA genotypes to modify the association between sexual abuse and MAOA first exon CpG and mean methylation?}

Table 2 presents the results from regression models predicting exon 1 mean methylation and methylation at $\mathrm{CpG} 2 / 3$, $4,5 / 6,7 / 8,10,11$, and 15 that included three predictors: $M A O A$ genotype, SA, and the interaction of MAOA genotype and SA. Results indicated that genotype was predictive of methylation levels at CpGs 2/3, 4, 10, and mean exon 1 methylation levels. SA was predictive of methylation levels at $\mathrm{CpG} 2 / 3,4,5 / 6,10$, and mean exon 1 methylation levels. 


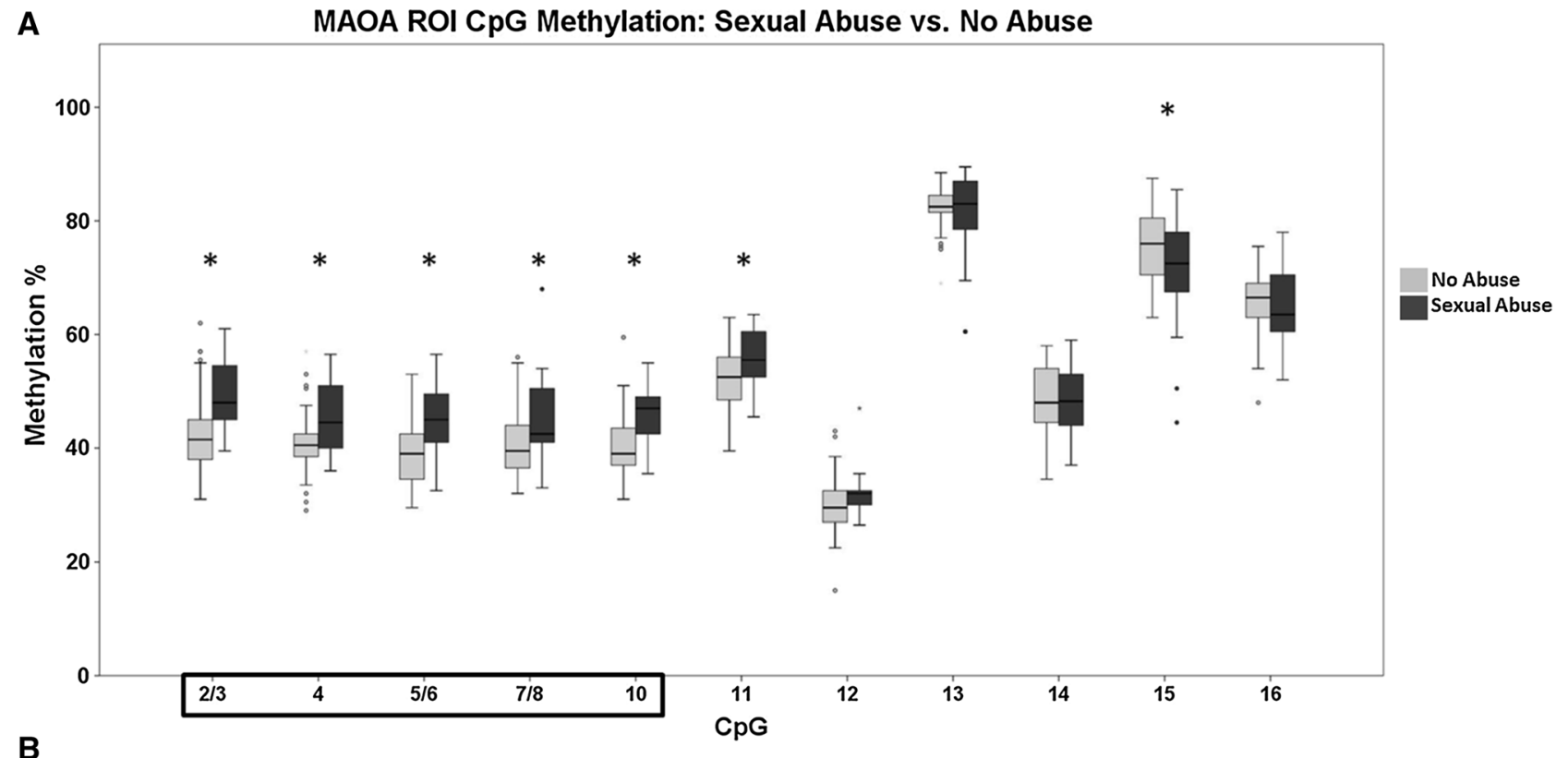

B

MAOA Region of Interest CpG Sites


GCATTCAGGTCAGTGTGGAC $\mathbf{C G}^{\mathbf{1 1}}$ TAG $_{\text {CG }} \mathbf{1 2}_{\text {GTGGCCTGGGGGACCCTGGCCAGTGAGGGGTAGGGGAACCTACAGTAGCTCTTGTGGTGTTGGGGGTCTCTCATGCATGCG }}{ }^{\mathbf{1 3}}$ AGAGT GTAGTGTAGCCATGGCTTGGCCCCATATCCTGCG ${ }^{\mathbf{1 4}}$ AGGTAGGAGTGGGGGTTGTGCCAGTITGCTGGTGGTGTGACTGGGGGAGGCAGACACAATAATITACTACTACTACTATTAAATAC

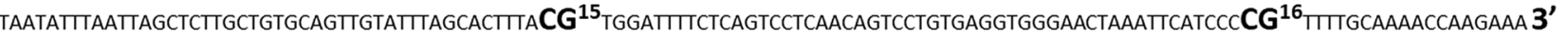

Fig. 1 a Results of comparisons of methylation levels across the $M A O A$ ROI CpGs $(* p<0.05)$ observed among women who had and who had not experienced sexual abuse. No data were available for

\section{Does MAOA first exon methylation mediate associations of sexual abuse with alcohol dependence, drug dependence, depression disorders, anxiety disorders, and CD?}

Significantly greater proportions of participants who experienced SA than NA presented both lifetime and current diagnoses of alcohol dependence, drug dependence, anxiety disorders, and CD prior to age 15 (see Online Resource Table S2). In addition, a greater proportion of participants who experienced SA than of the non-abused presented lifetime depressive disorders, while the proportions with the current depression were similar.

Results of significant final step-wise models testing whether methylation mediated associations of SA with diagnoses are presented in Table 3 and all results in Online Resource Tables S3 and S4. Mediation was detected only for increased risk of current depression. Methylation at CpGs $2 / 3,4,5 / 6,7 / 8$, and exon 1 mean levels mediated the association between SA and current depression. As presented in Table 3, methylation at CpGs $2 / 3,4,5 / 6,7 / 8$, and of exon 1 fully mediated the association of SA and depression. Lifetime depressive disorders were predicted
CpGs 1 and 9. b Genomic sequence of the MAOA ROI with all $\mathrm{CpG}$ sites enlarged, bolded, and numbered, and with the exonic protein coding region sequence underlined

independently by SA and methylation at $\mathrm{CpG} 5 / 6$. By contrast, lifetime alcohol dependence was predicted only by SA.

\section{Post hoc analyses}

Women with current depression $(n=46)$ as compared to women with no lifetime or current depression $(n=45)$ presented higher levels of methylation at $\mathrm{CpGs}, 2 / 3$ $[t(88)=2.63, p=0.010], 5 / 6[t(88)=2.98, p=0.004], 7 / 8$ $[t(88)=2.70, p=0.008], 10[t(88)=2.04, p=0.004], 11$ $[t(88)=1.99, p=0.050]$, and $12[t(88)=2.00, p=0.049]$, as well as mean methylation levels of the first exon $[t(88)=2.81, p=0.006]$ and ROI $[t(88)=3.23, p=0.002]$. Among the women with current depression, a higher level of methylation was associated with past or current use of any medication (stimulants, hypnotics, anxiolytics, antidepressants, and antipsychotics) at $\mathrm{CpG} 7 / 8[t(34)=2.04$, $p=0.049$ ]. No differences in methylation were observed when antidepressants were considered alone. Women with current or lifetime diagnoses of panic disorder $(n=36)$ as compared to women with no current or lifetime diagnoses of anxiety or depression $(n=48)$ presented higher levels of methylation of the first exon $[t(70)=3.43, p=0.001]$ 
Table 2 Results of regression models predicting $M A O A$ Exon 1 methylation by $M A O A$ genotype and sexual abuse

$\underline{\text { General-linear model } \text { Observed power }}$ $\overline{d f \quad F \quad p}$

$\mathrm{CpG} 2 / 3$

MAOA-uVNTR ${ }^{\mathrm{a}}$

Sexual abuse

MAOA-uVNTR $\times$ sexual abuse

Adjusted $R^{2}=0.186$

CpG4

\section{MAOA-uVNTR}

Sexual abuse

MAOA-uVNTR $\times$ sexual abuse

Adjusted $R^{2}=0.146$

CpG5/6

\begin{tabular}{|c|c|c|c|c|}
\hline MAOA-uVNTR & 1 & 4.507 & 0.105 & 0.131 \\
\hline Sexual abuse & 1 & 17.029 & $<0.001$ & 0.974 \\
\hline $\begin{array}{l}\text { MAOA-uVNTR } \times \text { sexual } \\
\text { abuse }\end{array}$ & & 2.856 & 0.240 & 0.275 \\
\hline \multicolumn{5}{|l|}{ Adjusted $R^{2}=0.197$} \\
\hline \multicolumn{5}{|l|}{ CpG7/8 } \\
\hline MAOA-uVNTR & 1 & 1.888 & 0.389 & 0.192 \\
\hline Sexual abuse & 1 & 8.522 & 0.004 & 0.788 \\
\hline $\begin{array}{l}\text { MAOA-uVNTR } \times \text { sexual } \\
\text { abuse }\end{array}$ & & 0.506 & 0.776 & 0.085 \\
\hline \multicolumn{5}{|l|}{ Adjusted $R^{2}=0.068$} \\
\hline \multicolumn{5}{|l|}{ CpG10 } \\
\hline MAOA-uVNTR & 1 & 10.583 & 0.005 & 0.546 \\
\hline Sexual abuse & 1 & 16.276 & $<0.001$ & 0.968 \\
\hline $\begin{array}{l}\text { MAOA-uVNTR } \times \text { sexual } \\
\text { abuse }\end{array}$ & & 0.412 & 0.814 & 0.078 \\
\hline \multicolumn{5}{|l|}{ Adjusted $R^{2}=0.206$} \\
\hline \multicolumn{5}{|l|}{ Exon 1 mean methylation } \\
\hline MAOA-uVNTR & 1 & 7.188 & 0.027 & 0.262 \\
\hline Sexual abuse & 1 & 20.097 & $<0.001$ & 0.989 \\
\hline $\begin{array}{l}\text { MAOA-uVNTR } \times \text { sexual } \\
\text { abuse }\end{array}$ & & 1.223 & 0.542 & 0.139 \\
\hline \multicolumn{5}{|l|}{ Adjusted $R^{2}=0.214$} \\
\hline \multicolumn{5}{|l|}{ CpG11 } \\
\hline MAOA-uVNTR & 1 & 4.662 & 0.097 & 0.306 \\
\hline Sexual abuse & 1 & 6.452 & 0.011 & 0.670 \\
\hline $\begin{array}{l}\text { MAOA-uVNTR } \times \text { sexual } \\
\text { abuse }\end{array}$ & & 0.018 & 0.991 & 0.051 \\
\hline \multicolumn{5}{|l|}{ Adjusted $R^{2}=0.072$} \\
\hline \multicolumn{5}{|l|}{ CpG15 } \\
\hline MAOA-uVNTR & 1 & 1.108 & 0.575 & 0.058 \\
\hline Sexual abuse & 1 & 1.971 & 0.160 & 0.264 \\
\hline $\begin{array}{l}\text { MAOA-uVNTR } \times \text { sexual } \\
\text { abuse }\end{array}$ & & 0.936 & 0.626 & 0.117 \\
\hline Adjusted $R^{2}=0.004$ & & & & \\
\hline
\end{tabular}

${ }^{a}$ Monoamine oxidase A variable number of tandem repeats and ROI $[t(70)=3.33, p=0.001]$, as well as at CpGs $2 / 3$ $[t(70)=3.64, p=0.001], 4[t(70)=2.10, p=0.040], 5 / 6$ $[t(70)=3.65, p=0.001], 7 / 8[t(70)=2.29, p=0.025]$, and $10[t(70)=2.88, p=0.005]$.

\section{Discussion}

Among young women, SA was associated with hypermethylation of the MAOA ROI and particularly the first exonic region of $M A O A$. This association was robust to adjustments for factors common among individuals who have experienced maltreatment, including psychoactive medication, alcohol and drug dependence, and current use of alcohol and drugs. The first exonic region contains binding domains for transcription factors associated with transcriptional enhancement (See Online Resource Table S5). First exon methylation of genes is suggested to play a stronger role in transcriptional silencing through the blockade of transcript initiation, compared to methylation in upstream promoter loci which contributes to up or downregulation of transcriptional activity (Brenet et al. 2011). Methylation of the region flanking the MAOA first exon has been associated with MAO enzymatic activity in the brain (Shumay et al. 2012), transcriptional downregulation in vitro, and with 5-HT levels in whole blood (Checknita et al. 2015). In a recent study, higher methylation levels specific to the MAOA first exon were observed in women (mean 45.3\%) compared to men (mean 13.7\%) (Melas and Forsell 2015). Here, we report a similar level of methylation at MAOA exon 1 (mean 44.3\%, SD 5.9) in women. Thus, the higher levels of MAOA first exon methylation observed among women are indicative of sites involved in X-chromosome inactivation of one of the two MAOA alleles by DNA methylation (Cotton et al. 2011), further suggesting a functional role of methylation in this region. While the study focused primarily on the well-characterized first exonic region of $M A O A$, it is noteworthy that SA was also associated with hypomethylation of intronic CpG 15, 260-bp downstream from the first exonic region. Similar to increased exonic methylation, lower intronic methylation also typically associates with transcriptional downregulation (Jones 2012; Moore et al. 2013). As such, altered intronic methylation of MAOA among those who experienced SA may have also contributed to the associations reported here.

While SA was consistently associated with hypermethylation of the MAOA ROI, similar results regarding PA were less clear due to the small number of participants who experienced PA only. The previous studies of methylation in this region among females also reported no association with a measure of adversity defined as parental death, divorce, financial problems, or other family problems (Melas et al. 2013; Melas and Forsell 2015). 
Table 3 Factors associated with current and lifetime disorders

\begin{tabular}{|c|c|c|c|c|c|c|c|}
\hline \multirow[t]{2}{*}{ Predictor } & \multicolumn{2}{|l|}{ Step 1} & \multicolumn{2}{|l|}{ Step 2} & \multicolumn{2}{|l|}{ Step 3} & \multirow{2}{*}{$\begin{array}{l}\text { Mediation analysis } \\
\text { Bootstrapped } 95 \% \\
\text { confidence interval }\end{array}$} \\
\hline & Odds ratio & $\begin{array}{l}95 \% \\
\text { confidence } \\
\text { interval }\end{array}$ & Odds ratio & $\begin{array}{l}95 \% \\
\text { confidence } \\
\text { interval }\end{array}$ & Odds ratio & $\begin{array}{l}95 \% \\
\text { confidence } \\
\text { interval }\end{array}$ & \\
\hline \multicolumn{8}{|c|}{ Lifetime depression disorders } \\
\hline Sexual abuse & 5.60 & $1.95-16.04$ & & & 3.37 & $1.08-10.54$ & \\
\hline CpG5/6 & & & 1.14 & $1.05-1.24$ & $\begin{array}{l}1.10 \\
\chi^{2}(1, N=75)=15.30, p<0.001\end{array}$ & $1.01-1.21$ & - \\
\hline \multicolumn{8}{|c|}{ Current depression disorders } \\
\hline Sexual abuse & 5.60 & $1.95-16.04$ & & & 1.20 & $0.38-3.80$ & \\
\hline $\mathrm{CpG} 2 / 3$ & & & 1.11 & $1.03-1.20$ & $\begin{array}{l}1,11 \\
\chi^{2}(1, N=75)=8.77, p=0.012\end{array}$ & $1.02-1.20$ & $0.13-1.33$ \\
\hline Sexual abuse & 5.60 & $1.95-16.04$ & & & 1.38 & $0.44-4.26$ & \\
\hline CpG4 & & & 1.11 & $1.02-1.21$ & $\begin{array}{l}1.10 \\
\chi^{2}(1, N=75)=6.05, p=0.049\end{array}$ & $1.00-1.21$ & $1.33-1.42$ \\
\hline Sexual abuse & 5.60 & $1.95-16.04$ & & & 1.01 & $0.30-3.35$ & \\
\hline CpG5/6 & & & 1.14 & $1.04-1.25$ & $\begin{array}{l}1.14 \\
\chi^{2}(1, N=75)=9.96, p=0.007\end{array}$ & $1.04-1.26$ & $0.16-2.01$ \\
\hline Sexual abuse & 5.60 & $1.95-16.04$ & & & 1.40 & $0.46-4.29$ & \\
\hline $\mathrm{CpG} 7 / 8$ & & & 1.11 & $1.02-1.20$ & $\begin{array}{l}1.10 \\
\chi^{2}(1, N=75)=7.10, p=0.029\end{array}$ & $1.01-1.19$ & $0.04-1.22$ \\
\hline Sexual abuse & 5.60 & $1.95-16.04$ & & & 1.02 & $0.31-3.39$ & \\
\hline Exon 1 & & & 1.16 & $1.05-1.28$ & $\begin{array}{l}1.16 \\
\chi^{2}(1, N=75)=9.74, p=0.008\end{array}$ & $1.04-1.29$ & $0.17-1.22$ \\
\hline
\end{tabular}

${ }^{a}$ Mediation analysis conducted using bootstrapped bias-corrected $95 \%$ confidence intervals with 5000 bootstrapping samples

First exon methylation was predicted by MAOA genotype and by SA, but not by the interaction of genotype and SA. These findings are consistent with a prior study that showed no association of $M A O A$ genotype with $M A O A$ methylation or brain MAO enzymatic activity in men (Shumay et al. 2012). Another study showed that reductions of panic disorder symptoms following cognitivebehavioural treatment were accompanied by MAOA exon 1 methylation levels similar to those of healthy women and that changes in methylation levels did not differ by genotype (Ziegler et al. 2016). MAOA-SS is associated with positive response to an antidepressant, mirtazapine in men (Tzeng et al. 2009), and to cognitive-behavioural therapy in men and women (Reif et al. 2014), and amygdala hyperreactivity in men (Meyer-Lindenberg et al. 2006) suggesting increased sensitivity to environmental factors. Yet, in the present study, MAOA genotype did not influence the effect of SA on methylation levels. Deficient expression of $M A O A$ occurs early in life leading to inhibited outgrowth of the serotonergic system and low basal serotonin levels (Nordquist and Oreland 2010; Booij et al. 2015), that when impacted by negative environmental factors later in life promote mental disorders (Byrd and Manuck 2014). The results of the present study, while preliminary and in need of replication, suggest that this effect occurs independently of methylation.

$M A O A$ exon 1 hypermethylation was shown to mediate associations between SA and current depression. In addition, SA and hypermethylation of the MAOA ROI were independently associated with lifetime depression. Although the observed mediation effects were small, this finding is consistent with the notion that many biological and environmental factors are involved in modulating associations between SA and depression (Labonte and Turecki 2012).

While the present study consistently showed that hypermethylation of the MAOA ROI was associated with depression, two previous studies of males and females reported hypomethylation in this same region among depressed women (Melas et al. 2013; Melas and Forsell 2015). The two previous studies included small samples with wide age ranges, a different measure of adversity, no measure of genotypes, and participants that may differ in comorbid disorders from the present sample. In the present study, almost all of the women with current depression had a long history of depression, and $37.7 \%$ had presented CD prior to age 15 . Depression is commonly comorbid with $\mathrm{CD}$ in adolescence (Angold et al. 1999), and adolescent CD predicts severe depression in adult women (Choi et al. 2016). We found 
that SA was directly associated with $\mathrm{CD}$, depression, and hypermethylation of the MAOA ROI. Given the previous evidence of hypermethylation of this same region among male offenders with ASPD, it may be that hypermethylation of this region of MAOA is associated specifically with depression among females with prior antisocial behaviour and experiences of SA, consistent with the notion that both the presentation and etiology of depression are heterogeneous (Januar et al. 2015).

Unfortunately, many young females experience SA, which as the results of the present study highlight, is associated with alcohol dependence, drug dependence, anxiety, depression, and CD. The present study shows that SA was associated with hypermethylation of MAOA exon 1 . Furthermore, hypermethylation of this region mediated the association of SA with current depression and was independently associated with lifetime depression. Elucidating neurobiological mechanisms by which SA promotes the development of mental disorders will provide a basis for the development of pharmacological and cognitive-behavioural interventions to limit the negative consequences of SA. The finding that reductions in symptoms of panic disorder following cognitive-behavioural treatment (Ziegler et al. 2016) were associated with a normalization of methylation levels underlines the importance of understanding the links between mental disorders and epigenetic mechanisms.

Robust evidence shows that MAOA genotype moderates the association between maltreatment and mental disorders (Wermter et al. 2010; Mandelli and Serretti 2013; Byrd and Manuck 2014), and recent evidence shows associations between MAOA methylation and mental disorders (Melas et al. 2013; Melas and Forsell 2015; Checknita et al. 2015; Ziegler et al. 2016). Furthermore, our study, and others (Melas et al. 2013; Melas and Forsell 2015; Ziegler et al. 2016), showed that genotype and environmental trauma independently associate with MAOA ROI methylation. Such findings, however, are preliminary and require replication. $M A O A$ genotype acts early in life to regulate, or set, lifelong levels of serotonergic functioning (Nordquist and Oreland 2010). The level of serotonergic functioning may increase risk of altered methylation, though our results suggest that it does so independently of environmental trauma. By contrast, environmental trauma, such as SA, altered methylation regardless of MAOA genotype. Given that methylation levels were more strongly associated with SA than with genotype in our study, it may be that proximal traumas have the greatest impact on methylation. It may be of importance that $M A O A$ methylation only mediated associations between SA and current depression, and not those with other disorders. This finding highlights the need for future studies exploring genotypes, epigenetics, environmental traumas, mental disorders, and the associations of various mental disorders with the interactions of these factors (Holz et al. 2014).

\section{Limitations and strengths}

As this was a preliminary and exploratory study with a relatively small sample, we lacked sufficient statistical power to perform multiple corrections for all of the statistical models used, and particularly for the regression models that included only participants with SA and two predictors. However, the initial group comparisons of methylation of maltreated and non-maltreated women used Fisher's LSD post hoc analyses with similar results obtained following more stringent Bonferroni corrections. These initial group comparisons used two-way mixed-model ANOVA models which included $\mathrm{CpG}$ as a repeated measure, thus allowing for the detection of group differences in overall methylation, and differences at each $\mathrm{CpG}$ corrected for multiple comparisons. As such, this approach offers a means to mitigate issues with multiple testing and has been adopted in the previous epigenetic studies (Labonte et al. 2012; Gross et al. 2013; Cruceanu et al. 2016).

The study design did not allow us to determine when alterations to methylation occurred, nor if it they were a direct consequence of maltreatment. Although our findings are in line with a large body of evidence associating maltreatment with altered methylation (Szyf 2011; Nagy and Turecki 2012), future prospective studies with repeated assessments of maltreatment and methylation are needed to determine if the association is direct and when the methylation changes occur. Type, severity, and chronicity of the maltreatment likely influence both the association and timing of the methylation changes (Szyf 2011; Nagy and Turecki 2012; Vachon et al. 2015). However, as summarized in Online Resource 1, analyses showed that women who had experienced one or both types of abuse exhibited increased exonic CpGs 1-10 and CpG 11 methylation levels relative to those who experienced no-abuse, with no differences between those who experienced one, or both, types of abuse at these same CpGs. No group differences in methylation for CpGs 12-16 were observed. These findings emphasize the importance of considering severity of abuse in future studies, particularly given that child victims often experience multiple types of abuse (Vachon et al. 2015). Few participants experienced PA only, thus limiting confidence in our results examining the association of PA only with methylation. In addition, data on nicotine use were not available. Smoking has been associated with altered methylation levels of $M A O A$ in healthy participants (Philibert et al. 2010), but two previous studies of clinical samples of women indicated no associations between smoking and methylation levels in the MAOA ROI (Melas et al. 2013; Melas and Forsell 2015).

DNA was extracted from saliva which contains a diverse range of peripheral cell-types, thus limiting the 
interpretation of our results in relation to central nervous system processes. However, a previous study identified an inverse correlation between MAOA promoter methylation in whole-blood DNA and MAO enzymatic activity in the brain (Shumay et al. 2012). In addition, methylation patterns observed in our study are consistent with methylation patterns in the MAOA ROI reported elsewhere in whole-blood samples in women (Melas et al. 2013; Melas and Forsell 2015; Ziegler et al. 2016). Thus, measurements of MAOA methylation in peripheral tissues may be viable proxies for central processes. Furthermore, as the EpiTYPER technique cannot distinguish methylation between $\mathrm{X}$-chromosome alleles, we were unable to examine X-chromosome inactivation. Given the potential importance of X-chromosome inactivation in the regulation of MAOA in women, its impact will be important to elucidate in subsequent epigenetic studies examining associations of abuse and mental disorders.

The study is also characterized by strengths including extensive data on mental disorders, antisocial behaviour, and maltreatment that were assessed with validated instruments. These rich data allowed us to determine that the observed association between SA and MAOA first exon methylation levels were robust to adjustment for lifetime and current use of psychoactive medications, lifetime and current diagnoses of alcohol and drug dependence, and current alcohol and drug use. It is important to note that these factors, among other environmental factors, may contribute to further alteration of MAOA methylation. Another strength of the study was the use of "gold-standard" genotyping and methylation analyses. Although self-reported measures of maltreatment involve a risk for information bias (Piquero et al. 2014), underreporting often biases reports from official records or other sources (Shaffer et al. 2008). Most participants reported on maltreatment in mid-adolescence and again in the early adulthood, as some participants who were afraid or reluctant to report earlier instances of maltreatment only did so in adulthood. In addition, although group comparisons of methylation levels between abused and non-abused groups were computed using post hoc LSD analyses, similar results were obtained using the more stringent Bonferroni formula to correct alpha levels.

\section{Conclusions}

We show that, in females, SA was associated with hypermethylation of the region spanning the MAOA first exon and part of the first intron. This association was robust to adjustments for lifetime and current psychoactive medication, alcohol and drug dependence, and current use of alcohol and drugs. MAOA genotype and SA, but not their interactions, were associated with $M A O A$ first exon methylation levels.
Although SA was directly associated with all mental disorders examined, hypermethylation of the MAOA first exon mediated the association between SA and current depression, and was directly associated with lifelong depression. Our results, along with the previous evidence, highlight the importance of epigenetic regulation of this region of $M A O A$ for furthering understanding of mechanisms underlying several different mental disorders that appear to depend, to some extent, on type of adversity or trauma experienced.

Acknowledgements The study was funded by grants from the Swedish Council for Working Life and Social Research, Mobilisering mot Narkotika, the county councils of Stockholm and Västmanland, and Karolinska Institutet Strategic Funding.

\section{Compliance with ethical standards}

Conflict of interest The authors report no biomedical financial interests or potential conflicts of interest.

Ethical approval All procedures performed in studies involving human participants were in accordance with the ethical standards of the institutional and/or national research committee and with the 1964 Helsinki declaration and its later amendments or comparable ethical standards. All waves of data collection were approved by the Regional Ethical Review Board in Stockholm. At each wave of data collection, participants signed informed consents, except at baseline when consent for those under age 18 was given by their parents. At collection of saliva samples all participants were 18 or older, and they specifically consented to providing saliva samples for DNA extraction. Confidentiality of all the information was guaranteed, and, at each wave of data collection, participants received gift certificates as compensation for their time.

Open Access This article is distributed under the terms of the Creative Commons Attribution 4.0 International License (http://creativeco mmons.org/licenses/by/4.0/), which permits unrestricted use, distribution, and reproduction in any medium, provided you give appropriate credit to the original author(s) and the source, provide a link to the Creative Commons license, and indicate if changes were made.

\section{References}

Alexander N, Wankerl M, Hennig J et al (2014) DNA methylation profiles within the serotonin transporter gene moderate the association of 5-HTTLPR and cortisol stress reactivity. Transl Psychiatry 4:e443. https://doi.org/10.1038/tp.2014.88

Angold A, Costello EJ, Erkanli A (1999) Comorbidity. J Child Psychol Psychiatry 40:57-87

Åslund C, Comasco E, Nordquist N et al (2013) Self-reported family socioeconomic status, the 5-HTTLPR genotype, and delinquent behavior in a community-based adolescent population. Aggress Behav 39:52-63. https://doi.org/10.1002/ab.21451

Babor T, Higgins-Biddle JC, Saunders JB, Monteiro MG (2001) The alcohol use disorders identification test: guidelines for use in primary care. Geneva World Health Org. https://doi. org/10.1177/0269881110393051

Beach SRH, Brody GH, Gunter TD et al (2010) Child maltreatment moderates the association of MAOA with symptoms of depression 
and antisocial personality disorder. J Fam Psychol 24:12-20. https ://doi.org/10.1037/a0018074

Berman AH, Bergman H, Palmstierna T, Schlyter F (2005) Evaluation of the drug use disorders identification test (DUDIT) in criminal justice and detoxification settings and in a Swedish population sample. Eur Addict Res 11:22-31. https://doi.org/10.1159/00008 1413

Booij L, Tremblay R, Szyf M, Benkelfat C (2015) Genetic and early environmental influences on the serotonin system: consequences for brain development and risk for psychopathology. J Psychiatry Neurosci 40:5-18. https://doi.org/10.1503/jpn.140099

Brenet F, Moh M, Funk P et al (2011) DNA methylation of the first exon is tightly linked to transcriptional silencing. PLoS One 6:e14524. https://doi.org/10.1371/journal.pone.0014524

Byrd AL, Manuck SB (2014) MAOA, childhood maltreatment, and antisocial behavior: meta-analysis of a gene-environment interaction. Biol Psychiatry 75:9-17. https://doi.org/10.1016/j.biops ych.2013.05.004

Checknita D, Maussion G, Labonté B et al (2015) Monoamine oxidase A gene promoter methylation and transcriptional downregulation in an offender population with antisocial personality disorder. Br J Psychiatry 206:216-222. https://doi.org/10.1192/ bjp.bp.114.144964

Choi TK, Worley MJ, Trim RS et al (2016) Effect of adolescent substance use and antisocial behavior on the development of early adulthood depression. Psychiatry Res 238:143-149. https://doi. org/10.1016/j.psychres.2016.02.036

Comai S, Tau M, Gobbi G (2012) The psychopharmacology of aggressive behavior: a translational approach: part 1: neurobiology. J Clin Psychopharmacol 32:83-94. https://doi.org/10.1097/ JCP.0b013e31823f8770

Cotton AM, Lam L, Affleck JG et al (2011) Chromosome-wide DNA methylation analysis predicts human tissue-specific $\mathrm{X}$ inactivation. Hum Genet 130:187-201. https://doi.org/10.1007/s0043 9-011-1007-8

Cruceanu C, Kutsarova E, Chen ES et al (2016) DNA hypomethylation of Synapsin II CpG islands associates with increased gene expression in bipolar disorder and major depression. BMC Psychiatry 16:286. https://doi.org/10.1186/s12888-016-0989-0

Dammann G, Teschler S, Haag T et al (2011) Increased DNA methylation of neuropsychiatric genes occurs in borderline personality disorder. Epigenetics 6:1454-1462. https://doi.org/10.4161/ epi.6.12.18363

Fergusson DM, Boden JM, Horwood LJ (2008) Exposure to childhood sexual and physical abuse and adjustment in early adulthood. Child Abuse Negl 32:607-619. https://doi.org/10.1016/j. chiabu.2006.12.018

Goldstein RB, Chou SP, Saha TD et al (2017) The epidemiology of antisocial behavioral syndromes in adulthood: results from the national epidemiologic survey on alcohol and related conditions-III. J Clin Psychiatry 78:90-98. https://doi.org/10.4088/ JCP. $15 \mathrm{~m} 10358$

Gross JA, Fiori LM, Labonté B et al (2013) Effects of promoter methylation on increased expression of polyamine biosynthetic genes in suicide. J Psychiatr Res 47:513-519. https://doi.org/10.1016/j. jpsychires.2012.11.016

Hayes AF (2013) Introduction to mediation, moderation, and conditional process analysis: a regression-based approach. Guilford Press, New York

Herringa RJ, Birn RM, Ruttle PL et al (2013) Childhood maltreatment is associated with altered fear circuitry and increased internalizing symptoms by late adolescence. Proc Natl Acad Sci 110:1911919124. https://doi.org/10.1073/pnas.1310766110

Hill J, Nathan R (2008) Childhood antecedents of serious violence in adult male offenders. Aggress Behav 34:329-338. https://doi. org/10.1002/ab.20237
Hodgins S, De Brito SA, Chhabra P, Côté G (2010) Anxiety disorders among offenders with antisocial personality disorders: a distinct subtype? Can J Psychiatry 55:784-791

Holz N, Boecker R, Buchmann AF et al (2014) Evidence for a sexdependent MAOA $\times$ childhood stress interaction in the neural circuitry of aggression. Cereb Cortex 26:1-11. https://doi. org/10.1093/cercor/bhu249

Hussey JM, Chang JJ, Kotch JB (2006) Child maltreatment in the United States: prevalence, risk factors, and adolescent health consequences. Pediatrics 118:933-942. https://doi.org/10.1542/ peds.2005-2452

Januar V, Saffery R, Ryan J (2015) Epigenetics and depressive disorders: a review of current progress and future directions. Int J Epidemiol 44:1364-1387. https://doi.org/10.1093/ije/dyu273

Jones PA (2012) Functions of DNA methylation: islands, start sites, gene bodies and beyond. Nat Rev Genet 13:484-492

Kooiman CG, Ouwehand AW, ter Kuile MM (2002) The Sexual and Physical Abuse Questionnaire (SPAQ). A screening instrument for adults to assess past and current experiences of abuse. Child Abuse Negl 26:939-953

Koss MP, Oros CJ (1982) Sexual experiences survey: a research instrument investigating sexual aggression and victimization. J Consult Clin Psychol 50:455-457. https://doi. org/10.1037/0022-006X.50.3.455

Labonte B, Turecki G (2012) Epigenetic effects of childhood adversity in the brain and suicide risk. In: Dwivedi Y (ed) The Neurobiologcial basis of suicide, CRC Press/Taylor \& Francis, Boca Raton

Labonte B, Yerko V, Gross J et al (2012) Differential glucocorticoid receptor exon 1(B), 1(C), and $1(\mathrm{H})$ expression and methylation in suicide completers with a history of childhood abuse. Biol Psychiatry 72:41-48. https://doi.org/10.1016/j.biops ych.2012.01.034

Lenzenweger MF, Lane MC, Loranger AW, Kessler RC (2007) DSMIV personality disorders in the national comorbidity survey replication. Biol Psychiatry 62:553-564. https://doi.org/10.1016/j. biopsych.2006.09.019

Li M, Arcy CD, Meng X (2016) Maltreatment in childhood substantially increases the risk of adult depression and anxiety in prospective cohort studies: systematic review, meta- analysis, and proportional attributable fractions. Psychol Med 46:717-730. https://doi.org/10.1017/S0033291715002743

Mandelli L, Serretti A (2013) Gene environment interaction studies in depression and suicidal behavior: an update. Neurosci Biobehav Rev 37:2375-2397. https://doi.org/10.1016/j.neubi orev.2013.07.011

McCrory E, De Brito SA, Viding E (2010) Research review: the neurobiology and genetics of maltreatment and adversity. J Child Psychol Psychiatry 51:1079-1095. https://doi.org/10.111 1/j.1469-7610.2010.02271.x

McCrory E, De Brito SA, Viding E (2012) The link between child abuse and psychopathology: a review of neurobiological and genetic research. J R Soc Med 105:151-156. https://doi. org/10.1258/jrsm.2011.110222

Melas PA, Forsell Y (2015) Hypomethylation of MAOA's first exon region in depression: a replication study. Psychiatry Res 226:2-4. https://doi.org/10.1016/j.psychres.2015.01.003

Melas PA, Wei Y, Wong CCY et al (2013) Genetic and epigenetic associations of MAOA and NR3C1 with depression and childhood adversities. Int J Neuropsychopharmacol 16:1513-1528. https:// doi.org/10.1017/S1461145713000102

Meyer-Lindenberg A, Buckholtz JW, Kolachana B et al (2006) Neural mechanisms of genetic risk for impulsivity and violence in humans. Proc Natl Acad Sci USA 103:6269-6274. https://doi. org/10.1073/pnas.0511311103 
Moore LD, Le T, Fan G (2013) DNA methylation and its basic function. Neuropsychopharmacology 38:23-38. https://doi. org/10.1038/npp.2012.112

Nagy C, Turecki G (2012) Sensitive periods in epigenetics: bringing us closer to complex behavioral phenotypes. Epigenomics 4:445457. https://doi.org/10.2217/epi.12.37

Nestler EJ (2014) Epigenetic mechanisms of drug addiction. Neuropharmacology 76:1-22. https://doi.org/10.1016/j.neuropharm .2013.04.004.Epigenetic

Nordquist N, Oreland L (2010) Serotonin, genetic variability, behaviour, and psychiatric disorders-a review. Ups J Med Sci 115:210. https://doi.org/10.3109/03009730903573246

Pavlov KA, Chistiakov DA, Chekhonin VP (2012) Genetic determinants of aggression and impulsivity in humans. J Appl Genet 53:61-82. https://doi.org/10.1007/s13353-011-0069-6

Philibert RA, Gunter TD, Beach SR et al (2008) MAOA methylation is associated with nicotine and alcohol dependence in women. Am J Med Genet 0:1-9. https://doi.org/10.1002/ajmg.b.30778.MAOA

Philibert RA, Beach SRH, Gunter TD et al (2010) The effect of smoking on MAOA promoter methylation in DNA prepared from lymphoblasts and whole blood. Am J Med Genet 0:619-628. https ://doi.org/10.1002/ajmg.b.31031

Piquero AR, Schubert CA, Brame R (2014) Comparing official and self-report records of offending across gender and race/ethnicity in a longitudinal study of serious youthful offenders. Crim Behav Ment Health 25:112-125. https://doi.org/10.1002/cbm.1914

Reif A, Richter J, Straube B et al (2014) MAOA and mechanisms of panic disorder revisited: from bench to molecular psychotherapy. Mol Psychiatry 19:122-128. https://doi.org/10.1038/mp.2012.172

Rita M, Reichl C, Parzer P et al (2016) Associations between depression and specific childhood experiences of abuse and neglect: a meta-analysis. J Affect Disord 190:47-55. https://doi. org/10.1016/j.jad.2015.09.006

Sabol SZ, Hu S, Hamer D (1998) A functional polymorphism in the monoamine oxidase A gene promoter. Hum Genet 103:273-279

Shaffer A, Huston L, Egeland B (2008) Identification of child maltreatment using prospective and self- report methodologies: a comparison of maltreatment incidence and relation to later psychopathology. Child Abuse Negl 32:682-692. https://doi.org/10.1038/ nrm 2621

Shumay E, Fowler JS (2010) Identification and characterization of putative methylation targets in the MAOA locus using bioinformatic approaches. Epigenetics 5:325-342

Shumay E, Logan J, Volkow ND, Fowler JS (2012) Evidence that the methylation state of the monoamine oxidase A (MAOA) gene predicts brain activity of MAO A enzyme in healthy men. Epigenetics 7:1151-1160. https://doi.org/10.4161/epi.21976

Singh MM, Parsekar SS, Nair SN (2014) An epidemiological overview of child sexual abuse. J Fam Med Prim Care 3:430-435. https:// doi.org/10.4103/2249-4863.148139

Springer KW, Sheridan J, Kuo D, Carnes M (2007) Long-term physical and mental health consequences of childhood physical abuse: results from a large population-based sample of men and women. Child Abuse Negl 31:517-530. https://doi.org/10.1016/j.chiab u. 2007.01.003.Long-term

Steadman HJ, Mulvey EP, Monahan J et al (1998) Violence by people discharged from acute psychiatric inpatient facilities and by others in the same neighborhoods. Arch Gen Psychiatry 55:393-401

Straus MA, Hamby SL, Boney-McCoy S, Sugarman DB (1996) The Revised Conflict Tactics Scales. J Fam Issues 17:283-316. https ://doi.org/10.1177/019251396017003001

Szyf M (2011) DNA methylation, the early-life social environment and behavioral disorders. J Neurodev Disord 3:238-249. https://doi. org/10.1007/s11689-011-9079-2

Szyf M (2015) Prospects for the development of epigenetic drugs for CNS conditions. Nat Rev Drug Discov 14:461-474. https://doi. org/10.1038/nrd4580

Teicher MH, Samson JA (2016) Annual research review : enduring neurobiological effects of childhood abuse and neglect. J Child Psychol Psychiatry 3:241-266. https://doi.org/10.1111/jcpp.12507

Thompson MP, Kingree JB, Desai S (2004) Gender differences in long-term health consequences of physical abuse of children : data from a nationally representative survey. Am J Public Health 94:599-604

Tzeng D-S, Chien C-C, Lung F-W, Yang C-Y (2009) MAOA gene polymorphisms and response to mirtazapine in major depression. Hum Psychopharmacol 24:293-300. https://doi.org/10.1002/hup

Vachon DD, Krueger RF, Rogosch FA, Cicchetti D (2015) Assessment of the harmful psychiatric and behavioral effects of different forms of child maltreatment. JAMA Psychiatry 55455:1135-1142. https ://doi.org/10.1001/jamapsychiatry.2015.1792

Wermter AK, Laucht M, Schimmelmann BG et al (2010) From nature versus nurture, via nature and nurture, to gene $\times$ environment interaction in mental disorders. Eur Child Adolesc Psychiatry 19:199-210. https://doi.org/10.1007/s00787-009-0082-z

Ziegler C, Richter J, Mahr M et al (2016) MAOA gene hypomethylation in panic disorder-reversibility of an epigenetic risk pattern by psychotherapy. Transl Psychiatry 6:e773. https://doi.org/10.1038/ tp.2016.41 